\title{
DECOMPOSABILITY OF COMPLETELY SUSLIN-ADDITIVE FAMILIES
}

\author{
ZDENĚK FROLIK AND PETR HOLICKY
}

\begin{abstract}
It is proved that every point-finite completely Suslin-additive family in the product of a complete metric space by a compact space is $\sigma$-discretely decomposable. Several applications are indicated.
\end{abstract}

The main result is in Theorem 1 which simultaneously generalizes the Hansell lemma [Ha [K-P, Theorem 1], and also a particular case of $\left[F_{1}\right.$, Theorem 1] saying that a metrizable Baire measurable image of an analytic space (here $\omega$-analytic) is separable.

The main results are stated in $\S 1$, various examples and possible applications are sketched in $\$ 2$, and the proofs of Lemma 1 and Theorem 1 are left for $\$ 3$.

It should be noted that Theorem 2, the main application below, is seemingly the deepest result in the generalization of the theory of $\omega$-analytic spaces (called $K$-analytic by $\mathrm{G}$. Choquet $\left.\left[\mathrm{Ch}_{1}\right]-\left[\mathrm{Ch}_{2}\right]\right)$ to the nonseparable case, generalizing the Hansell extension to metric spaces. The basic concepts and results are explained in $\left[\mathbf{F}-\mathbf{H}_{3}\right]-\left[\mathbf{F}-\mathbf{H}_{4}\right]$. It should be remarked that some results were already announced in $\left[\mathbf{F}-\mathbf{H}_{1}\right]-\left[\mathbf{F}-\mathbf{H}_{2}\right]$ and $\left[\mathbf{F}_{2}\right]$ and in a communication by the second author at the 5 th Prague Topological Symposium, 1976.

1. Results. For simplicity, by a topological space we mean a Hausdorff completely regular space, and by a uniform space a separated uniform space. The main result reads:

THEOREM 1. Let $f$ be an usco-compact correspondence from a complete metric space $M$ into a topological space $X$. If $\left\{X_{a} \mid a \in A\right\}$ is a point-finite completely Suslin-additive family in $X$, then the family $\left\{f^{-1}\left[X_{a}\right] \mid a \in A\right\}$ is o-discretely decomposable (abbreviated $\sigma-d d$ ) in $M$.

Here usco-compact means upper semicontinuous with the "values" compact. Recall that a Suslin set in a topological space $X$ is a subset in $X$ which admits a Suslin representation by closed sets, i.e. a set of the form $\cup\left\{\cap_{n=0}^{\infty} F_{\sigma \mid n+1} \mid \sigma \in \omega^{\omega}\right\}$ where $F_{o \mid n+1}$ is closed in $X$. A certain familiarity with the Suslin operation is assumed, see $\$ 3$.

Received by the editors November 19, 1979 and, in revised form, April 14, 1980 and June 19, 1980. 1980 Mathematics Subject Classification. Primary 28A05, 54H05; Secondary 54C60, 54D30, 54E15, $54 \mathrm{E} 50$. 
A family $\left\{X_{a}\right\}$ is completely $\Re$-additive if the union of each subfamily of $\left\{X_{a}\right\}$ is in $\mathfrak{N}$. Here this notion is used only when $\mathfrak{N}$ is the collection of all Suslin sets or all Baire sets in a space.

Finally, a family $\left\{X_{a} \mid a \in A\right\}$ is called $\sigma$-discretely decomposable, abbreviated $\sigma$-dd, if there exists a family $\left\{X_{a n} \mid a \in A, n \in \omega\right\}$ such that each $\left\{X_{a n} \mid a \in A\right\}$ is discrete, and $X_{a}=\cup\left\{X_{a n} \mid n \in \omega\right\}$ for each $a$ in $A$. In a uniform space, as in a metric space, e.g. in Theorem 1 , discrete is understood in the uniform sense. In a topological space "discrete" has its usual meaning. Of course we may also consider "discrete" in the sense of the fine uniformity, i.e. discrete means discrete with respect to some continuous pseudometric, and this concept of discrete is used for defining analytic topological space.

A correspondence from a uniform space $X$ into a uniform space $Y$ is called $\sigma$-dd-preserving if for each discrete, or equivalently $\sigma$-dd, family $\left\{X_{a}\right\}$ in $X$ the family $\left\{f\left[X_{a}\right]\right\}$ is $\sigma$-dd in $Y$. A uniform space is called analytic if there exists an usco-compact $\sigma$-dd preserving correspondence from a complete metric space $M$ onto $A$; if the weight of $M$ is $\kappa>\omega$ then $A$ is called $\kappa$-analytic. A topological space is called $\kappa$-analytic if the fine uniformity is $\kappa$-analytic. Now Theorem 1 implies immediately:

THEOREM 2. In an analytic space every point-finite completely Suslin-additive family is $\sigma-d d$.

REMARK. It is easy to see that, in any space, every $\sigma$-dd family of Suslin sets is completely Suslin-additive.

Note that a correspondence from a space with discrete families countable is $\sigma$-dd-preserving. Hence $\omega$-analytic is just "analytic" as used by many authors [S], $\left[F_{1}\right]-\left[F_{4}\right],[R]$, and called $K$-analytic by $G$. Choquet. Note also that spaces called analytic here were called hyperanalytic in previous papers by the present authors $\left[\mathbf{F}-\mathbf{H}_{\mathbf{1}}\right]-\left[\mathbf{F}-\mathbf{H}_{2}\right],\left[\mathbf{F}_{2}\right],[\mathbf{H o}]$.

It is self-evident that every complete metric space is analytic; in this case Theorem 2 is just the Kaniewski-Pol [K-P] generalization of the original result of Hansell in $\left[\mathrm{Ha}_{1}\right]$ (where the family is assumed disjoint).

It is easy to show that the product of a complete metric space $M$ by a compact space $K$ is also analytic (consider the inverse of the projection $M \times K \rightarrow M$ ).

The basic theory of analytic spaces will appear in two papers $\left[\mathrm{F}-\mathrm{H}_{3}\right]-\left[\mathrm{F}-\mathrm{H}_{4}\right]$; the theory generalizes both the "separable" theory of $\omega$-analytic spaces as well as the Hansell theory in metric spaces $\left[\mathrm{Ha}_{2}\right],\left[\mathrm{Ha}_{3}\right]$. For the proof of Theorem 1 we shall use Hansell's method, and the following particular case of Theorem 1, which concerns $\omega$-analytic spaces.

LEMMA 1. In an w-analytic space, in particular in a compact space, each point-finite completely Suslin-additive family is countable.

For the proof of Lemma 1 we shall use the following result from $\left[F_{1}\right]$, or rather an obvious corollary to that stated below. 
LEMMA 2. In an w-analytic space, in particular, in a compact space, each disjoint completely Suslin-additive family is countable.

Proof. By [ $F_{1}$, Theorem 1] if a Baire measurable image $X$ of an $\omega$-analytic space is metrizable then $X$ is separable. Now let $\mathscr{P}$ be a disjoint completely Suslinadditive family of nonvoid sets in an $\omega$-analytic space $A$. The union $Y$ of $\mathscr{P}$ is also $\omega$-analytic space. Let $f$ be the projection of $Y$ onto $\mathscr{P}$, and let $\mathscr{P}$ have the trivial metric. It follows from the first separation principle that $f$ is Baire measurable. Hence $\mathcal{P}$ is countable.

COROllaRY TO LEMMA 2. In any w-analytic space, in particular in any compact space, every star-countable completely Suslin-additive family is countable.

Proof. The family of "connected" components of the family is disjoint, and each of them is the union of a countable number of elements of the family.

2. Remarks. Call a uniform space Hansell (or Hansell-pf) if every completely Suslin-additive family which is disjoint (or point-finite) is $\sigma$-dd. A topological space is called Hansell or Hansell-pf if the fine uniformity is Hansell or Hansell-pf. A topological space is called topologically Hansell or topologically Hansell-pf if it enjoys the above property with "discrete" in the topological sense. Note that in a paracompact space the two kinds of discreteness coincide.

2.1. Examples. (a) Theorem 2 says that every analytic space is Hansell-pf.

(b) No $Q$-set is Hansell. (Recall that a $Q$-set is an uncountable subspace $X$ of the reals such that each subset of $X$ is $F_{\sigma}$.)

(c) Fleissner [FI] showed that if there exists a supercompact cardinal then there is a model such that each metric space is Hansell.

(d) An example of a topologically Hansell-pf space which is not Hansell. Consider any pseudocompact space $X$ which is $\sigma$-discrete with discrete understood in the topological sense, e.g. $X \subset \beta \omega, X=\omega \cup D, D$ isolated and each infinite subset of $\omega$ has a cluster point in $D$.

(e) An absolute example of a paracompact space which is not Hansell. Let $X$ be the real line with the topology such that $U \subset X$ is open iff $U=V \cup Y$ where $V$ is open in $R$ in the usual topology, and $Y \subset R-Q$. It is easy to see that $\{\{x\} \mid x \in$ $X\}$ is completely Suslin-additive. On the other hand $R-Q$ is not $F_{\sigma}$ in $X$ because if $R-Q=\cup\left\{F_{n} \mid n \in \omega\right\}$ then the interior of $R-F_{n}$ in $R$ contains $Q$, and this contradicts the Baire Category Theorem in $R$.

2.2. Applications. (a) Let $f$ be a Baire measurable mapping of a Hansell topological space $X$ into a metric space (Baire sets form the smallest $\sigma$-algebra making all continuous functions measurable). Then $f$ has a class $\left(\left[\mathbf{F}_{5}\right],\left[\mathbf{P}_{1}\right]\right.$, see also $\left.\left[\mathbf{F}_{2}\right]\right)$. Further applications in this spirit can be found in $\left[\mathbf{F}_{\mathbf{6}}\right],\left[\mathbf{F}_{\mathbf{7}}\right]$.

(b) A selection theorem. Let $X$ be a Hansell-pf topological space and $Y$ a metric space. Then every compact-valued mapping $F: X \rightarrow 2^{Y}$ of lower Baire class $\alpha$, where $0<\alpha<\omega_{1}$, admits a selector of class $\alpha$. The proof of [K-P, Theorem 2] works. 
(c) Let $f$ be a compact-valued correspondence from a Hansell-pf space $X$ into a topological space $T$ such that the preimages of Suslin sets are Suslin. If $\left\{T_{a}\right\}$ is $\sigma$-dd in $T$, then $\left\{f^{-1}\left[T_{a}\right]\right\}$ is $\sigma$-dd in $X$. In particular, if $T$ is a Suslin subset in $X$ then the embedding $T \hookrightarrow X$ is $\sigma$-dd-preserving. Hence, if $g: X \rightarrow Y$ is a $\sigma$-dd-preserving correspondence, $X$ is Hansell, and $T$ is Suslin in $X$, then $g: T \rightarrow Y$ is $\sigma$-dd-preserving. Note the case when $X$ is analytic.

(d) Let $X$ and $Y$ be Hansell spaces with the same Suslin sets. Then they have the same $\sigma$-dd families.

2.3. Analytic spaces. One can easily see that every analytic topological space is paracompact. Call a topological space $A$ topologically analytic if in the definition the discreteness is considered in the topological sense. Topologically analytic spaces do not need to be paracompact, e.g. 2.1(d); however they are topologically Hansell-pf (Theorem 1). The fundamental properties of topologically analytic spaces will be studied elsewhere.

3. Proofs. In fact we shall work with Suslin sets as the images of $\omega^{\omega}$ under the closed-graph correspondences. What we need is the following "Suslin stratification" of Suslin sets.

Every Suslin set $K$ in a space can be written

$$
K=\bigcup\left\{\bigcap\left\{K_{\sigma \mid n+1} \mid n \in \omega\right\} \mid \sigma \in \omega^{\omega}\right\}
$$

such that

(1) $\cap\left\{K_{\sigma \mid n+1} \mid n \in \omega\right\}=\bigcap\left\{\bar{K}_{\sigma \mid n+1} \mid n \in \omega\right\}$ for each $\sigma \in \omega^{\omega}$,

(2) $K_{\sigma_{0}, \ldots, \sigma_{n}}=\cup\left\{K_{\sigma_{0}} \ldots, \sigma_{n}, i \mid i \in \omega\right\}$,

(3) all $K_{\sigma \mid n+1}$ are Suslin sets.

Indeed, if $K=\cup\left\{\cap\left\{F_{\sigma \mid n+1} \mid n \in \omega\right\} \mid \sigma \in \omega^{\omega}\right\}$ with $F_{\sigma \mid n+1}$ closed, it is enough to put

$$
K_{\sigma \mid k+1}=\bigcup\left\{\bigcap\left\{F_{\tau \mid n+1} \mid n \in \omega\right\}\left|\tau \in \omega^{\omega}, \sigma\right| k+1=\tau \mid k+1\right\} .
$$

In what follows, if any letter stands for a Suslin set, then the same letter indexed with a finite sequence is a member of such a fixed "Suslin stratification".

Proof of Lemma 1. Let $\left\{K_{a} \mid a \in A\right\}$ be an uncountable point-finite completely Suslin-additive family of nonvoid sets in a compact space (every $\omega$-analytic space can be embedded into a compact space, and every $\omega$-analytic space is Suslin in every space).

By induction we shall construct distinct indices $a_{j}$ in $A$ and $\sigma^{j} \in \omega^{\omega}, j \in \omega$, such that

$\left(\mathrm{P}_{p}\right)$ the set $Y_{p}=K_{a_{0}}\left(\sigma^{0} \mid p+1\right) \cap \cdots \cap K_{a_{p}}\left(\sigma^{p} \mid 1\right)$ intersects uncountably many $K_{a}$.

This would contradict the point-finiteness of $\left\{K_{a}\right\}$ because $Y_{p} \subset K_{a,}$ for $j=$ $0, \ldots, p ; \cap Y_{p}=\cap \bar{Y}_{p}$ because we are working with Suslin stratification, and finally $\cap \bar{Y}_{p} \neq \varnothing$ because the space is compact.

The construction goes as follows. By the corollary to Lemma 2 there exists $a_{0}$ such that $K_{a_{0}}$ meets uncountably many members of $\left\{K_{a} \mid a \in A\right\}$. There is a natural number $\sigma_{0}^{0}$ such that $\left(\mathrm{P}_{0}\right)$ holds, because the Suslin sets $\left\{K_{a_{0}}(i) \mid i \in \omega\right\}$ form a 
countable cover of $K_{a_{0}}$. Assuming that $a_{0}, \ldots, a_{p},\left(\sigma_{0}^{0}, \ldots, \sigma_{p}^{0}\right)=\sigma^{0} \mid p+$ $1, \ldots, \sigma_{0}^{p}=\sigma^{p} \mid 1$ are constructed with $\left(\mathrm{P}_{p}\right)$ satisfied, choose $a_{p+1}$ such that $Y_{p} \cap$ $K_{a_{p+1}}$ meets uncountably many members of $\left\{K_{a} \mid a \in A\right\}$. It is possible because the system $\left\{Y_{p} \cap K_{a} \mid a \in A \backslash\left\{a_{0}, \ldots, a_{p}\right\}\right\}$ is uncountable and Suslin-additive and it is therefore possible to use the corollary to Lemma 2 again.

The system $\left\{K_{a_{0}}\left(\sigma^{0} \mid p+1, i_{0}\right) \cap \cdots \cap K_{a_{p+1}}\left(i_{p+1}\right) \mid\left(i_{0}, \ldots, i_{p+1}\right) \in \omega^{p+2}\right\}$ forms a countable cover of the Suslin set $Y_{p} \cap K_{a_{p+1}}$. Hence we can find a finite sequence $\left(\sigma_{p+1}^{0}, \ldots, \sigma_{0}^{p+1}\right) \in \omega^{p+2}$ such that $\left(P_{p+1}\right)$ holds with respect to the preceding fact. The induction is finished.

Proof of Theorem 1. Assume that $\left\{f^{-1}\left[X_{a}\right] \mid a \in A\right\}$ is not $\sigma$-dd in $M$. Using the following two lemmas we shall construct a compact subset $C$ of $M$ (homeomorphic to $2^{\omega}$ ) such that the compact set $D=f[C]$ in $X$ ( $f$ is usco-compact) meets an uncountable number of $X_{a}$ 's, and this will contradict Lemma 1. For convenience we say that a family $\left\{Y_{a}\right\}$ in $M$ is not $\sigma$-dd in a point $m \in M$ if $\left\{Y_{a} \cap U\right\}$ is not $\sigma$-dd in $U$ for each neighbourhood $U$ of $\mathbf{m}$.

LemMA 3. Let $\left\{Y_{a} \mid a \in A\right\}$ be a point-countable family in the metric space $M$, which is not $\sigma$-dd. Then, there are two distinct points $m_{0}, m_{1}$ in $M$ such that $\left\{Y_{a} \mid a \in A\right\}$ is not $\sigma-d d$ in any of them.

LeMma 4. Let $\left\{Y_{a} \mid a \in A\right\}$ be a (point-countable) family of Suslin subsets of the (complete) metric space $M$, which is not $\sigma$-dd. Then there are two disjoint subsets $A^{0}$ and $A^{1}$ of $A$ such that $\left\{Y_{a} \mid a \in A^{j}\right\}$ is not $\sigma$-dd for $j=0,1$.

The lemmas are slight generalizations of $\left[\mathrm{Ha}_{1}\right.$, Lemmas 8,9$]$, and are proved in [K-P].

We shall construct open sets $U_{i \mid p+1} \subset M$ (denote $V_{i \mid p+1}=f\left[U_{i \mid p+1}\right]$ ) and sets $X^{i \mid p+1} \subset X, X_{a}^{i \mid p+1} \subset X_{a}, A^{i \mid p+1} \subset A$, natural numbers $\sigma_{0}^{i \mid p+1}, \ldots, \sigma_{p}^{i \mid p+1}$ (equivalently $\left.\sigma^{i \mid p+1} \mid p+1\right)$ in the $p$ th step of induction such that the following conditions hold for all $i \in\{0,1\}^{\omega}$.

(ip) diameter $U_{i \mid p+1} \leqslant(p+1)^{-1}$, and $U_{i \mid p+1} \subset U_{i \mid p}$ for $p>1$.

(ii $\bar{U}_{i \mid p, 0} \cap \bar{U}_{i \mid p, 1}=\varnothing$, where $U_{i \mid 0} \equiv U$.

(iii ${ }_{p} A^{i \mid p, 0} \cap A^{i \mid p, 1}=\varnothing$, where $A^{i \mid 0} \equiv A$.

$\left(\mathrm{iv}_{p}\right) X^{i \mid p+1}=\cup\left\{X_{a}^{i \mid p} \cap \bar{V}_{i \mid p+1} \mid a \in A^{i \mid p+1}\right\} \neq \varnothing$, where $X_{a}^{i \mid 0}=X_{a}$.

$\left(\mathrm{v}_{p}\right) X_{a}^{i \mid p+1}=\left.X_{a}^{i \mid p} \cap X_{\sigma}^{i \mid 1}\right|_{p+1} \mid p+1 \cap \cdots \cap X_{\sigma}^{i \mid p_{p+1}^{+1}}$.

$\left(\mathrm{vi}_{p}\right)\left\{f^{-1}\left[X_{a}^{i \mid p+1}\right] \cap U_{i \mid p+1} \mid a \in A^{i \mid p+1}\right\}$ is not $\sigma$-dd.

(vii $\left.{ }_{p}\right)\left\{X_{a}^{i \mid p+1} \mid a \in A^{i \mid p+1}\right\}$ is a point-finite completely Suslin-additive system.

For $p=0$ we proceed as follows: Consider $\left\{Y_{a}=f^{-1}\left[X_{a}\right] \mid a \in A\right\}$. Choose two disjoint sets $A^{0}, A^{1}$ as in Lemma 4. Then, we find $m_{0}, m_{1}$ as in Lemma 3, where $A^{0}$ stands instead of $A$, and further we find $m^{\prime}$ such that $\left\{Y_{a} \mid a \in A^{1}\right\}$ is not $\sigma$-dd in $m^{\prime}$ using Lemma 3 again. One of the pairs $\left(m_{0}, m^{\prime}\right)$ and $\left(m_{1}, m^{\prime}\right)$ can be separated by $U_{0}, U_{1}$ such that $\left(\mathrm{i}_{0}\right)$ and $\left(\mathrm{ii}_{0}\right)$ hold. By $\left(\mathrm{iv}_{0}\right)$ the Suslin sets $X^{i \mid 1}$ are defined. Since $\left\{f^{-1}\left[X_{n_{0}^{i \mid 1}} \cap X_{a}\right] \cap U_{i \mid 1} \mid n_{0}^{i \mid 1} \in \omega\right\}$ is a countable cover of $f^{-1}\left[X^{i \mid 1} \cap X_{a}\right] \cap U_{i \mid 1}=$ $f^{-1}\left[X_{a}\right] \cap U_{i \mid 1}$ for $a \in A^{i \mid 1}$, we find $\sigma_{0}^{i \mid 1}$ such that $\left\{f^{-1}\left[X_{a}^{i \mid 1}\right] \cap U_{i \mid 1}\right\}$ is not $\sigma$-dd for $i \in\{0,1\}^{\omega}$. Thus, $\left(\mathrm{vi}_{0}\right)$ holds and $\left(\mathrm{vii}_{0}\right)$ is trivial. 
The induction step is done in the same way (consider $X_{a}^{i \mid p}$ instead of $X_{a}, A^{i \mid p}$ instead of $A$ ).

Now the set $\cap\left\{\overline{X^{i \mid p+1}} \mid p \in \omega\right\}$ is a nonempty compact subset of $f\left(c_{i}\right)$, where $\left\{c_{i}\right\}=\cap\left\{\bar{U}_{i \mid p+1} \mid p \in \omega\right\}$, because

$$
\bigcap\left\{\overline{X^{i \mid p+1}} \mid p \in \omega\right\} \subset \bigcap\left\{\overline{f\left[U_{i \mid p+1}\right]} \mid p \in \omega\right\}=f\left(c_{i}\right) .
$$

The last equality follows from the fact that $f$ is usco-compact. Further

$$
\bigcap\left\{\overline{X^{i \mid p+1}} \mid p \in \omega\right\} \subset X^{i \mid p_{0}+1} \subset \bigcup\left\{X_{a} \mid a \in A^{i \mid p_{0}+1}\right\}
$$

for every $p_{0} \in \omega$. The sets $C=\cup\left\{\cap\left\{\bar{U}_{i \mid p+1} \mid p \in \omega\right\} \mid i \in\{0,1\}^{\omega}\right\}$ and $D=f[C]$ are compact, because $f$ is an upper semicontinuous compact-valued correspondence.

Let us identify the sets $C$ and $\{0,1\}^{\omega}$, and define $\varphi(d)=\left\{a \in A \mid d \in X_{a}\right\}$ for $d \in D$. Suppose that the set $\cup\{\varphi(d) \mid d \in D\}$ is countable. Denote by $Z_{K}$ the set $\left\{i \in C \equiv\{0,1\}^{\omega} \mid \exists\left(d \in \cap\left\{X^{i \mid p+1} \mid p \in \omega\right\}\right)\right.$ s.t. $\left.\varphi(d)=K\right\}$ for finite $K \subset$ $\cup\{\varphi(d) \mid d \in D\}$. Using (iii ${ }_{p}$ ) for every $p \in \omega$ and (+) we prove that $Z_{K}$ has at most as many elements as $K$.

To prove the mentioned assertion suppose that $i^{1}, \ldots, i^{l}$ are distinct elements of $Z_{K}, d^{j} \in \cap\left\{\overline{X^{j^{j} \mid p+1}} \mid p \in \omega\right\}, \varphi\left(d^{j}\right)=K$, and $i^{j} \mid t+1$ are distinct for suitable $t \in \omega, j=1, \ldots, l$. According to $(+)$ we have $f\left(i^{j}\right) \cap X_{a_{j}} \ni d^{j}$ for some $a_{j} \in$ $A^{i / t+1}\left(f\left(i^{j}\right) \equiv f\left(c_{i}\right)\right)$. We found $l$ distinct elements of $K\left(a_{j}\right.$ are distinct according to (iii $)_{\text {)). }}$

Thus $Z_{K}$ is finite, and $Z=\cup\left\{Z_{K} \mid K\right.$ is a finite subset of $\left.\cup\{\varphi(d) \mid d \in D\}\right\}$ is countable. Since $C$ is uncountable, we find an $i_{0} \in C \backslash Z$ and $d_{0} \in f\left(i_{0}\right)$ such that $i_{0} \in Z_{\varphi\left(d_{0}\right)}$, and this is a contradiction. Thus the set $\cup\{\varphi(d) \mid d \in D\}$ is uncountable, and $D \cap K_{a} \neq \varnothing$ for uncountably many $a \in A$. This contradicts Lemma 1 and Theorem 1 is proved.

\section{REFERENCES}

[Ch$h_{1}$ G. Choquet, Ensembles $K$-analytique et $K$-Souslin, Ann. Inst. Fourier (Grenoble) 9 (1959), 77-81.

$\left[\mathrm{Ch}_{2}\right] \ldots$, Theory of capacities, Ann. Inst. Fourier (Grenoble) 4(1953/1954), 131-294.

[FI] W. G. Fleissner, An axiom for nonseparable Borel theory, Trans. Amer. Math. Soc. 251 (1979), 309-328.

[F $F_{1}$ Z. Frolik, A measurable map with analytic domain and metrizable range is quotient, Bull. Amer. Math. Soc. 76 (1970), 1112-1117.

$\left[\mathrm{F}_{2}\right] \ldots$, The concept of non-separable analytic set, Colloq. Math. Soc. János Bolyai 23, Topology, Budapest, Hungary, 1978, pp. 449-461.

$\left[\mathrm{F}_{3}\right] \longrightarrow, \mathrm{A}$ contribution to the descriptive theory of sets and spaces, General Topology and its Relations to Modern Analysis and Algebra, Academia, Praha, 1962, pp. 157-173.

[F] _ Analytic and Borelian sets in general spaces, Proc. London Math. Soc. 21 (1970), 674-692.

[F $F_{5}$, Interplay of measurable and uniform spaces, Topology and its Applications, Proc. Internat. Sympos. on Topology and its Applications (Budva, 1972), Beograd, 1973, pp. 98-101.

[F $\mathbf{F}_{6}$, Baire sets and uniformities on complete metric spaces, Comment. Math. Univ. Carolin. 13 (1972), 137-147.

[ $\left[\mathrm{F}_{7}\right] \_$Four functors into paved spaces, Seminar Uniform Spaces (1973-1974), Matematický Ústav CSAV, Praha, 1975, pp. 27-72.

[F-H $]$ Z. Frolík and P. Holický, o-discrete decomposability of completely additive families, Seminar Uniform Spaces (1975-1976), Matematický Ústav ĊAV, Praha, 1976. 
[F- $\left.\mathrm{H}_{2}\right] \_$On the non-separable descriptive theory, Fifth Winter School on Abstract Analysis, Matematický Ústav CSAV, Praha, 1977.

[F- $\left.\mathrm{H}_{3}\right]$ _ Analytic and Luzin spaces (non-separable case), preprint.

$\left[\mathbf{F}-\mathbf{H}_{4}\right] \ldots$, Consequences of Luzin separation principle (non-separable case), preprint.

$\left[\mathrm{Ha}_{1}\right]$ R. W. Hansell, Borel measurable mappings for nonseparable metric spaces, Trans. Amer. Math. Soc. 161 (1971), 145-169.

[Ha $]$, On the non-separable theory of $k$-Borel and $k$-Suslin sets, General Topology Appl. 3 (1973), 161-195.

[Ha $\left.\mathrm{Ha}_{3}\right]$, On characterizing non-separable analytic and extended Borel sets as types of continuous images, Proc. London Math. Soc. 28 (1974), 683-699.

[Ho] P. Holický, Neseparabilni analytické prostory (Non-separable analytic spaces), CSc. thesis, Charles University, 1977.

[K-P] J. Kaniewski and R. Pol, Borel-measurable selectors for compact-valued mappings in the non-separable case, Bull. Acad. Polon. Sci. Sér. Sci. Math. 23 (1975), 1043-1050.

$\left[\mathbf{P}_{1}\right]$ D. Preiss, Completely additive disjoint system of Baire sets is of bounded class, Comment. Math. Univ. Carolin. 15 (1972), 341-344.

$\left[\mathbf{P}_{2}\right] \ldots$ Baire class of completely additive systems of Baire sets, Seminar Uniform Spaces (1976-1977), Matematický Ústav CSAV, Praha, 1977.

[R] C. A. Rogers, Analytic sets in Hausdorff spaces, Mathematica 11 (1964), 1-8.

[S] M. Sion, On analytic sets in topological spaces, Trans. Amer. Math. Soc. 96 (1960), 341-345.

Mathematical Institute, ČSAV, 11567 Prague 1, Czechoslovakia

Mathematical Department, Research and Test aeronautical Institute, 19905 Prague, Czechoslovakia 